\title{
CONDITIONAL BANACH SPACES, CONDITIONAL PROJECTIONS AND GENERALIZED MARTINGALES
}

\author{
NGUYEN-XUAN-LOC and EGGERT BRIEM
}

\section{Introduction.}

Given a probability space $(\Omega, \mathcal{B}, P)$, and a sub- $\sigma$-field $\mathcal{B}^{\prime}$ of $\mathcal{B}$, it is well known that the restriction of the conditional expectation to $L^{2}(B)$ is identical to the orthogonal projection from the Hilbert space $L^{2}(B)$ onto the sub-Hilbert-space $L^{2}\left(\mathcal{B}^{\prime}\right)$.

In this hilbertian case, the limit-martingale theorem is closely connected with the limit-theorem of an increasing sequence (with respect to the natural order) of bounded, symmetric operators. (See [2].)

The aim of this note is to give an operator-theoretic approach to martingale theory. Main results are:

The existence of the conditional expectation on $L^{1}(B)$ can be deduced from the linear aspects of the given structure (Theorem 1 and Theorem 3). Therefore it is natural to consider the problem on a class of Banach spaces which we call CB-spaces (Definitions 1 and 3).

If we look only for a $L^{1}$-limit of the martingale, the analytic proof given here (Theorem 2) avoids completely the use of Doob's well-known estimation on the "downcrossing number" of paths, which is deeply connected with measure theory.

This abstract approach when applied again to concrete spaces of probability theory covers well-known results of real-valued martingales and also a special case of vector-valued martingales (Examples 1 and 3).

From the functional analysis point of view, Theorem 2 gives a generalization of Vigier's result to a class of projections in Banach spaces which are even non-reflexive.

A summary of a part of this note has appeared in [1].

The first author wishes to express his gratitude to professor K. Ito, who has graciously supervised his study the past two years. Both authors wish to thank professor E. Thue Poulsen for many suggestions and improvements given by him after reading the manuscript.

Received May 1969; in revised form October 31, 1969. 


\section{GB-spaces and G-projections.}

Definition 1. A conditional Banach space (abbreviated CB-space) is a couple $(H,\|\cdot\|)$ where

1) $H$ is a real Hilbert space with scalar product $(\cdot, \cdot)$,

2) $\|\cdot\|$ is a norm on $H$ defining a topology which is coarser than the topology defined by $(\cdot, \cdot)$.

Let $X$ denote the Banach space obtained by completing $H$ under $\|\cdot\|$. Then we have a continuous injection

$$
(H,(\cdot, \cdot)) \subset(X,\|\cdot\|) \text {. }
$$

The transpose of this injection defines a map from $X^{*}$ into $H^{*}$. Composing with the canonical map from $H^{*}$ onto $H$ we get a map $\varphi$ from $X^{*}$ into $H$. It is given by

$$
\left(h, \varphi\left(x^{*}\right)\right)=\left\langle h, x^{*}\right\rangle \quad \text { for all } h \in H .
$$

Since $H$ is dense in $X$, the map $\varphi$ is an injection, and identifying $X^{*}$ with its image, we have

$$
X^{*} \subset H \subset X \text {. }
$$

REMaRK. We shall frequently use the notation $\left(X, H, X^{*}\right)$ for the CBspace $(H,\|\cdot\|)$.

Example 1. Let $(\Omega, \mathcal{B}, P)$ be a probability space. Then $L^{2}(\mathcal{B})$ with the norm induced by the $L^{1}$-norm $\|\cdot\|_{1}$ is a CB-space. The completion of $L^{2}(\mathcal{B})$ is in this case $L^{1}(\mathcal{B})$ itself.

Now let $H_{1}$ be a closed subspace of $H$ and let $X_{1}$ be the closure of $H_{1}$ under $\|\cdot\|$. Then $X_{1}$ is a closed subspace of the Banach space $X$. Just as above there exists for all $x_{1}^{*} \in X_{1}{ }^{*}$ an element $\varphi_{1}\left(x_{1}{ }^{*}\right)$ in $H_{1} \leqq H$ given by

$$
\left(h, \varphi_{1}\left(x_{1}^{*}\right)\right)=\left\langle h, x_{1}^{*}\right\rangle \text { for all } h \in H_{1} \text {. }
$$

We now consider the following condition:

Condition 1. For all $x_{1}{ }^{*} \in X_{1}{ }^{*}$, the linear form $\bar{x}_{1}{ }^{*}$ on $H$ defined by

$$
\left\langle h, \bar{x}_{1}^{*}\right\rangle=\left(h, \varphi_{1}\left(x_{1}^{*}\right)\right)
$$

is continuous on $H$ under the norm $\|\cdot\|$. Its continuous extension to $X$ is then also denoted $\bar{x}_{1}^{*}$.

This condition gives us a well defined map from $X^{*}{ }_{1}$ into $X^{*}$, and we have the following commutative scheme 


$$
\begin{aligned}
& X \gg H \gg X^{*} \\
& \stackrel{\vartheta}{X_{1}} \stackrel{\vartheta}{H_{1}} \gg \stackrel{\hat{\vdots}_{1}}{*} \text {. }
\end{aligned}
$$

We are interested in the case where the orthogonal projection from $H$ onto $H_{1}$ extends to a continuous projection from $X$ onto $X_{1}$.

Definition 2. If the orthogonal projection $P$ from $H$ onto $H_{1}$ extends to a continuous projection $\bar{P}$ from $X$ onto $X_{1}$, we shall call $H_{1}$ a sub-CB-space of $(H,\|\cdot\|)$. We shall also call $\left(X_{1}, H_{1}, X_{1}{ }^{*}\right)$ a sub-CB-space of $\left(X, H, X^{*}\right)$. The projection $\bar{P}$ is called the conditional projection (C-projection) of $X$ onto $X_{1}$.

We shall prove that $\left(X_{1}, H_{1}, X_{1}^{*}\right)$ is a sub-CB-space of $\left(X, H, X^{*}\right)$ if and only if Condition 1 is satisfied and the above defined map from $X_{1}{ }^{*}$ into $X^{*}$ is continuous (Theorem 1), and that every closed subspace $H_{1}$ of $H$, for which Condition 1 is satisfied is a sub-CB-space of $(H,\|\cdot\|)$, if $X$ is weakly sequentially complete (Theorem 3 ).

Theorem 1. $\left(X_{1}, H_{1}, X_{1}{ }^{*}\right)$ is a sub-CB-space of $\left(X, H, X^{*}\right)$ if and only if Condition 1 is satisfied and the map from $X_{1}{ }^{*}$ into $X^{*}$ is continuous. Furthermore, the conditional projection $\bar{P}$ has norm one, if and only if the map from $X_{1}^{*}$ into $X^{*}$ is an isometry.

Proof. Assume that the map from $X_{1}{ }^{*}$ into $X^{*}$ is continuous and of norm $c$, let $x_{1}{ }^{*} \in X_{1}{ }^{*}$ and let $\bar{x}_{1}{ }^{*}$ be the corresponding element of $X^{*}$. Let $h \in H$. Then

But

$$
\begin{aligned}
\left|\left\langle P h, x_{1}{ }^{*}\right\rangle\right| & =\mid\left(P h, \varphi_{1}\left(x_{1}{ }^{*}\right)|=|\left(h, P \varphi_{1}\left(x_{1}{ }^{*}\right)|=|\left(h, \varphi_{1}\left(x_{1}{ }^{*}\right) \mid\right.\right.\right. \\
& =\left|\left\langle h, \bar{x}_{1}{ }^{*}\right\rangle\right| \leqq\|h\|\left\|\bar{x}_{1}{ }^{*}\right\|_{*} \leqq c\|h\|\left\|x_{1}{ }^{*}\right\|_{*} .
\end{aligned}
$$

$$
\|P h\|=\sup \left\{\left|\left\langle P h, x_{1}^{*}\right\rangle\right|: x_{1}^{*} \in X_{1}^{*},\left\|x_{1}^{*}\right\| \leqq 1\right\} .
$$

Therefore $\|P h\| \leqq c\|h\|$. Thus, $P$ is continuous under $\|\cdot\|$, and its continuous extension to $X$ obviously becomes a projection $\bar{P}$ of $X$ onto $X_{1}$.

If the map from $X_{1}{ }^{*}$ into $X^{*}$ is an isometry, then $c=1$, and it follows that $\bar{P}$ has norm 1 .

Now suppose $\bar{P}$ has norm $c>0$. Let $h \in H$. Then, as above,

$$
\left|\left\langle h, \bar{x}_{1}{ }^{*}\right\rangle\right|=\left|\left\langle P h, x_{1}{ }^{*}\right\rangle\right| \leqq\|P h\|\left\|x_{1}{ }^{*}\right\|_{*} \leqq c\|h\|\left\|x_{1}{ }^{*}\right\|_{*} \text {. }
$$

Since $H$ is dense in $X$, it follows that $\left\|\bar{x}_{1}{ }^{*}\right\|_{*} \leqq c\left\|x_{1}{ }^{*}\right\|_{*}$. If $c=1$, we see that $\left\|\bar{x}_{1}{ }^{*}\right\|_{*} \leqq\left\|x_{1}{ }^{*}\right\|_{*}$. The other inequality is trivial and we are done. 
REmarks. 1) The underlying ideas in the above proof come from the following scheme which is the "dual-scheme" of the one above:

$$
\begin{gathered}
X^{*} \subset H=H^{*} \rightarrow X^{* *} \\
\downarrow \\
\bar{X}_{1}^{*} \subset H_{1}=H_{1}^{*} \rightarrow X_{1}^{* *} .
\end{gathered}
$$

2) $\left\langle x, \bar{x}_{1}^{*}\right\rangle=\left\langle\bar{P} x, x_{1}^{*}\right\rangle$ for all $x \in X$ and for all $x_{1}^{*} \in X_{1}^{*}$. Thus

$$
\{x \in X \mid \bar{P} x=0\}=\left\{x \in X \mid\left\langle x, \bar{x}_{1}^{*}\right\rangle=0 ; \forall x_{1}^{*} \in X_{1}^{*}\right\} \text {. }
$$

Definition 3. When $\bar{P}$ has norm 1 , we say that $H_{1}$ is a strict sub-CBspace of $H$.

Example 2. Return to Example 1 and let $\mathcal{B}^{\prime}$ be a sub- $\sigma$-algebra of $\mathcal{B}$. Then $\left(L^{2}\left(\mathcal{B}^{\prime}\right),\|\cdot\|_{1}\right)$ is a sub-CB-space of $\left(L^{2}(\mathcal{B}),\|\cdot\|_{1}\right)$ and the conditional projection $\bar{P}_{1,2}$ from $L^{1}(\mathcal{B})$ onto $L^{1}\left(\mathcal{B}^{\prime}\right)$ is identical to the restriction of the conditional expectation $E\left(\cdot \mid \mathcal{B}^{\prime}\right)$ to $L^{1}(\mathcal{B})$. In fact, the restriction of $E\left(\cdot \mid \mathcal{B}^{\prime}\right)$ to $L^{2}(\mathcal{B})$ is the orthogonal projection of $L^{2}(\mathcal{B})$ onto $L^{2}\left(\mathcal{B}^{\prime}\right)$, and $E\left(\cdot \mid \mathcal{B}^{\prime}\right)$ is a continuous map from $L^{1}(\mathcal{B})$ onto $L^{1}\left(\mathcal{B}^{\prime}\right)$, and therefore we must have $E\left(\cdot \mid B^{\prime}\right)=\bar{P}_{1,2}$ by the uniqueness of $\bar{P}_{1,2}$.

Our present definition of conditional projections in the particular case of CB-spaces of function type has an advantage in the sense that we can firstly avoid the use of the Radon-Nikodým theorem and secondly avoid the use of the idea of changes of events which occurs frequently in probability theory.

Example 3. Let $(\Omega, \mathcal{B}, P)$ be a probability space, and let $\mathcal{H}$ be a separable Hilbert space. By $L^{1}(\mathcal{B}, \mathcal{H})$ we denote the set of strongly $\mathcal{B}$-measurable functions from $\Omega$ into $\mathcal{H}$ which are Bochner integrable relative to $P$ (identifying functions which are equal a.s.). For $1 \leqq p<\infty$, $L^{p}(\mathcal{B}, \mathcal{H})$ denotes the set of strongly $\mathcal{B}$-measurable functions from $\Omega$ into $\mathcal{H}$ for which

$$
\int_{\Omega}\|x(\omega)\|^{p} d P<\infty
$$

and $L^{\infty}(\mathcal{B}, \mathcal{H})$ the set of strongly $\mathcal{B}$-measurable functions $x$ for which $\|x(\omega)\|$ is bounded a.s.

On $L^{2}(\mathcal{B}, \mathcal{H})$ we define the inner product

$$
(f, g)=\int_{\Omega}\langle f(\omega), g(\omega)\rangle d P(\omega) \quad \text { for all } f, g \in L^{2}(\mathcal{B}, \mathcal{H}),
$$


where $\langle\cdot, \cdot\rangle$ denotes the inner product in $\mathcal{H}$.

Equipped with this inner product $L^{2}(\mathcal{B}, \mathcal{H})$ becomes a Hilbert space. Obviously $L^{2}(\mathcal{B}, \mathcal{H})$ is dense in $L^{1}(\mathcal{B}, \mathcal{H})$, and the $L^{1}$-topology restricted to $L^{2}(\mathcal{B}, \mathcal{H})$ is coarser than the topology defined by the inner product. Thus, $\left(L^{2}(\mathcal{B}, \mathcal{H}),\|\cdot\|_{1}\right)$ is a CB-space, where $\|\cdot\|_{1}$ denotes the $L^{1}$-norm.

Since $\mathcal{H}$ is a separable Hilbert space the dual space of $L^{1}(\mathcal{B}, \mathcal{H})$ is $L^{\infty}(\mathcal{B}, \mathcal{H})$. From this it follows easily that if $\mathcal{B}^{\prime}$ is a sub- $\sigma$-field of $B$, then $\left(L^{2}\left(\mathcal{B}^{\prime}, \mathcal{H}\right),\|\cdot\|_{1}\right)$ is a sub-CB-space of $\left(L^{2}(\mathcal{B}, \mathcal{H}),\|\cdot\|_{1}\right)$.

It is easy to see that the orthogonal projection of a simple function $f \in L^{2}(\mathcal{B}, \mathcal{H})$ on $L^{2}\left(\mathcal{B}^{\prime}, \mathcal{H}\right)$ is exactly $E\left(f \mid \mathcal{B}^{\prime}\right)$. By a standard argument on continuous extension, $\bar{P}$ is the conditional expectation, with respect to $\mathcal{B}$, for Hilbert space valued random variables.

Corollary 1. If $\left(X_{2}, H_{2}, X_{2}{ }^{*}\right)$ is a sub-CB-space of $\left(X_{1}, H_{1}, X_{1}{ }^{*}\right)$, then it is a sub-CB-space of $\left(X, H, X^{*}\right)$, and

$$
\bar{P}_{2}=\bar{P}_{12} \bar{P}_{1} \text {, }
$$

where $\bar{P}_{12}$ is the C-projection of $X_{1}$ onto $X_{2}$ and $\bar{P}_{1}$ and $\bar{P}_{2}$ are the C-projections of $X$ onto $X_{1}$ and $X_{2}$, respectively. Consequently, the restriction of $\bar{P}_{2}$ to $X_{1}$ is $\bar{P}_{12}$.

Proof. Obviously, $P_{2} h=P_{12} P_{1} h$ for $h \in H$, and hence, by continuity, $\bar{P}_{2} x=\bar{P}_{12} \bar{P}_{1} x$ for $x \in X$.

Remark. To illustrate the above result we look at the CB-spaces $\left(L^{1}(\mathcal{B}), L^{2}(\mathcal{B}), L^{\infty}(\mathcal{B})\right)$ and $\left(L^{1}\left(\mathcal{B}^{\prime}\right), L^{2}\left(\mathcal{B}^{\prime}\right), L^{\infty}\left(\mathcal{B}^{\prime}\right)\right)$. The C-projection is identical with the restriction of the conditional expectation $E\left(\cdot \mid \mathcal{B}^{\prime}\right)$ to $L^{1}(\mathfrak{B})$, and

1) $E\left(f \mid \mathcal{B}^{\prime}\right)=f$ for all $f$ in $L^{1}\left(\mathcal{B}^{\prime}\right)$,

2) $E\left(f \mid \vec{B}^{\prime \prime}\right)=E\left(E\left(f \mid \vec{B}^{\prime}\right) \mid \mathcal{B}^{\prime \prime}\right)$ for $\mathcal{B}^{\prime \prime} \subset \mathcal{B}^{\prime} \subset \mathcal{B}$.

In this example the map from $L^{\infty}\left(\mathcal{B}^{\prime}\right)$ into $L^{\infty}(B)$ is clearly an isometry. Therefore by Theorem 1,

$$
\int_{\Omega}\left|E\left(f \mid \mathcal{B}^{\prime}\right)\right| d P \leqq \int_{\Omega}|f| d P .
$$

These are well-known results in probability theory.

\section{Generalized martingales.}

Let $I$ be an interval on $R$ open at the right endpoint $t_{d}$, assume that to every $t$ in $I$ there is associated a strict sub-CB-space $\left(X_{t}, H_{t}, X_{t}^{*}\right)$ of a 
given CB-space $\left(X, H, X^{*}\right)$, in such a way that the family $\left(X_{t}, H_{t}, X_{t}^{*}\right)_{t \in I}$ is increasing to the right, i.e.: $\left(X_{s}, H_{s}, X_{s}{ }^{*}\right)$ is a strict sub-CB-space of $\left(X_{t}, H_{t}, X_{t}^{*}\right)$ for $t>s, t, s \in I$. By $H_{t_{d}}$ we denote the closure of $\bigcup_{t \in I} H_{t}$ in $H$.

Remark. We have assumed that the sub-CB-spaces are strict. If we instead assume that the projections $P_{t}$ of $H$ onto $H_{t}$ are uniformly bounded with respect to $\|\cdot\|$, we will get the same result with only a slight alteration of the proofs.

Proposition 1. $H_{t_{d}}$ is a strict sub-CB-space of $(H,\|\cdot\|)$ (actually the smallest one containing the family $\left.\left(H_{t}\right)_{t \in I}\right)$ and each $H_{t}$ is a strict sub-CBspace of $\left(H_{t_{d}},\|\cdot\|\right)$.

Proof. Following Theorem 1 we must prove that the orthogonal projection $P_{t_{d}}$ from $H$ onto $H_{t_{d}}$ and the orthogonal projections from $H_{t_{d}}$ onto $H_{t}, t \in I$, are $\|\cdot\|$-continuous and have norm 1 . Let $h \in H,\|h\|=1$, and let $\varepsilon>0$. There exists $t_{0} \in I$ and $h_{t_{0}} \in H_{t_{0}}$ such that $\left\|P_{t_{d}} h-h_{t_{0}}\right\|_{H}$ is so small that $\left\|P_{t_{d}} h-h_{t_{0}}\right\| \leqq \varepsilon$. Put $k=h_{t_{0}}+\left(I-P_{t_{d}}\right) h$, then $\|k\| \leqq 1+\varepsilon$. Since $\bar{P}_{t_{0}}$ is continuous with norm 1 , we have

and hence

$$
\left\|h_{t_{0}}\right\|=\left\|\bar{P}_{t_{0}} k\right\| \leqq 1+\varepsilon,
$$

$$
\left\|P_{t_{d}} h\right\| \leqq\left\|P_{t_{d}} h-h_{t_{0}}\right\|+\left\|h_{t_{0}}\right\| \leqq 1+2 \varepsilon,
$$

This implies $\left\|P_{t_{d}} h\right\| \leqq 1$, since the above inequalities hold for every $\varepsilon>0$.

Since for each $t \in I$, the orthogonal projection from $H_{t_{d}}$ onto $H_{t}$ is the restriction of $P_{t}$ (the orthogonal projection from $H$ onto $H_{t}$ ) to $H_{t_{d}}$, it is trivially $\|\cdot\|$-continuous and of norm 1 .

Lemma. Let $\bar{P}_{t}$ denote the C-projection of $X$ onto $X_{t}$. Then for every element $x$ in $X$,

$$
\mathrm{s}-\lim _{t \uparrow t_{d}} \bar{P}_{t} x=\mathrm{s}-\lim _{t \uparrow t_{d}} \bar{P}_{t}\left(\bar{P}_{t_{d}} x\right)=\bar{P}_{t_{d}} x,
$$

where $\mathrm{s}-\mathrm{lim}$ (strong limit) denotes the limit with respect to the norm $\|\cdot\|$.

Proof. For $t \geqq s, t, s \in I \cup t_{d}$ we have

$$
\bar{P}_{t}\left(\bar{P}_{s} x\right)=\bar{P}_{s} x \text { for all } x \in X .
$$

Thus we might as well assume $x \in X_{t_{d}}$. For $\varepsilon>0$, there exist then a $t_{0} \in I$ and a $x_{t_{0}} \in X_{t_{0}}$ such that

$$
\left\|x_{t_{0}}-x\right\|<\varepsilon
$$


For $t \geqq t_{0}$ we have by the assumption of strictness

$$
\left\|\bar{P}_{t}\left(x_{t_{0}}\right)-\bar{P}_{t}(x)\right\| \leqq\left\|x_{t_{0}}-x\right\| .
$$

But $\bar{P}_{t}\left(x_{t_{0}}\right)=x_{t_{0}}$ for $t \geqq t_{0}$, so

$$
\left\|x-\bar{P}_{t}(x)\right\| \leqq 2 \varepsilon \quad \text { for } t \geqq t_{0} .
$$

Definition 4. A generalized martingale defined on $I$ is a collection $\left\{x_{t}: t \in I\right\}$ of elements of $X$ such that

a) $x_{t} \in X_{t}$ for all $t \in I$,

b) $\bar{P}_{t s}\left(x_{t}\right)=x_{s}$ for all $t \geqq s, t, s \in I$, where $\bar{P}_{t s}$ denotes the C-projection of $X_{t}$ onto $X_{s}$.

Theorem 2. Let $\left\{x_{t}: t \in I\right\}$ be a generalized martingale defined on $I$. Then the three conditions are equivalent:

1) $\mathrm{s}-\lim _{t \uparrow t_{d}} x_{t}$ exists

2) there exists an $x_{t_{d}} \in X_{t_{d}}$ such that $x_{t}=\bar{P}_{t}\left(x_{t_{d}}\right)$ for all $t \in I$;

3) the subset $\left\{x_{t}: t \in I\right\}$ of $X$ is weakly relatively compact.

Proof. We first prove 1) $\Rightarrow 2$ ). Take $x$ such that $\mathrm{s}-\lim _{t \uparrow t_{d}} x_{t}=x$, take any $s \in I$. Then by continuity of $\bar{P}_{s}$,

$$
\text { s-lim }{ }_{t \uparrow t_{d}} \bar{P}_{s}\left(x_{t}\right)=\bar{P}_{s}(x) .
$$

But by b) of Definition 4 and by Corollary 1 ,

Thus

$$
\bar{P}_{s}\left(x_{t}\right)=x_{s} \quad \text { for } t \geqq s \text {. }
$$

$$
x_{s}=\bar{P}_{s}(x) \text {. }
$$

2) $\Rightarrow 3$ ). By Eberlein-Smulian's theorem it is enough to prove that every sequence extracted from $\left\{x_{t}: t \in I\right\}$ has a weakly convergent"subsequence. Thus, consider $\left\{x_{t_{n}}: t \in I\right\}$. Then at least one of the following two possibilities must occur:

(i) there exists a decreasing subsequence $t_{n_{i}}$ of indices;

(ii) there exists an increasing subsequence $t_{n_{i}}$ of indices.

Case (i): Look at the Hilbert space projections $P_{h_{i}}$. We have

that is,

$$
\left(P_{t_{i}} h, h\right) \geqq\left(P_{t_{n_{k}}} h, h\right) \quad \text { for } i \leqq k, h \in H_{t_{d}},
$$

$$
I \geqq P_{t_{n_{1}}} \geqq P_{t_{n_{2}}} \geqq \ldots \geqq P_{t_{n_{k}}} \geqq \ldots \geqq 0 \text {. }
$$


Thus, the sequence of projections converges strongly (with respect to the Hilbert space norm) to some projection $P_{0}$ (see [2]). Consequently, for every $h \in H$, the sequence $\left\{P_{h_{k}} h: k \in \mathrm{N}\right\}$ is convergent in $H$, and hence in $X$. In view of the uniform boundedness of the operators $\bar{P}_{t_{n_{k}}}$, the same is true for every $x \in X$. Since $x_{t_{n_{k}}}=\bar{P}_{t_{n_{k}}} x_{h_{n_{1}}}$, it follows that $x_{t_{n_{k}}}$ converges strongly and thus weakly.

Case (ii): It follows from the lemma that the sequence $x_{t_{j}}$ converges strongly, and hence weakly, in $X$ in case $t_{n_{j}} \uparrow t_{0}=t_{d}$. In case $t_{0}<t_{d}$, the restriction of $x_{t}$ to the set $\left\{t \in I \mid t<t_{0}\right\}$ is also a martingale, and the result follows.

3) $\Rightarrow 1$ ). Let $t_{n}$ be a sequence tending increasingly to $t_{d}$. According to the Eberlein-Smulian theorem there is a subsequence $t_{n_{i}}$ such that $x_{t_{i}}$ has a weak limit $x$ in $X$. Hence there exists a sequence $y_{k}$ where each term is a convex combination of the $x_{t_{n_{i}}}$ 's such that s-lim $y_{k}=x$. Thus

But

$$
\text { s- } \lim _{k} \bar{P}_{t_{1}} y_{k}=\bar{P}_{t_{n_{1}}} x \text {. }
$$

$$
y_{k}=\sum_{i=1}^{n_{k}} \alpha_{i} x_{t_{i}} \quad \text { where } \quad \sum_{i=1}^{n_{k}} \alpha_{i}=1, \alpha_{i} \geqq 0 \text {, }
$$

so

$$
\bar{P}_{t_{n_{1}}} y_{k}=x_{t_{n_{1}}} \text {, that is, } x_{t_{n_{1}}}=\bar{P}_{t_{n_{1}}} x \text {. }
$$

In the same manner we prove that $x_{t_{n_{2}}}=\bar{P}_{t_{n_{2}}} x$. Continuing we get $x_{t_{n_{i}}}=\bar{P}_{h_{i}} x$ for $i=1,2, \ldots$ By the lemma it follows that s-lim ${ }_{n_{i}} x_{i_{i}}=x$. Suppose $\left\{t_{m}\right\}$ is another sequence tending increasingly to $t_{d}$ such that

$$
\text { s-lim }{ }_{m_{j}} x_{t_{m_{j}}}=\bar{P}_{t_{d}} x^{\prime}
$$

For each $t_{n_{i}}$ there exists a $t_{m_{j}}>t_{n_{i}}$. Thus,

$$
x_{t_{n_{i}}}=\bar{P}_{t_{i}}\left(x_{t_{m_{j}}}\right)=\bar{P}_{t_{n_{i}}}\left(\bar{P}_{t_{m_{j}}}\left(x^{\prime}\right)\right)=\bar{P}_{t_{i}}\left(x^{\prime}\right),
$$

and then, according to the lemma,

whence

$$
\mathrm{s}-\lim _{n_{i}} x_{t_{i}}=\bar{P}_{t_{d}}\left(x^{\prime}\right)
$$

$$
x^{\prime}=\bar{P}_{t_{d}}\left(x^{\prime}\right)=\bar{P}_{t_{d}}(x)=x \text {. }
$$

A standard argument now shows that

$$
x=\mathrm{s}-\lim _{t \uparrow t_{d}} x_{t} .
$$

Remark. In the proof of 3) $\Rightarrow 1$ ) in Theorem 2, we can use directly the fact that every countable set of $\left\{x_{t}: t \in I\right\}$ has a weak limit point 
and that for fixed $t, P_{t}$ is weakly continuous, hence actually we do not have to use the stronger statement of the Eberlein-Smulian theorem used in the proof above.

Example 4. Let $\left\{\left(\mathcal{B}_{t}\right)\right\}_{t \in I}$ be an increasing family of sub- $\sigma$-algebras of $\boldsymbol{B}$. Then the closure in $L^{2}(\mathcal{B})$ of $\bigcup_{t \in I} L^{2}\left(\mathcal{B}_{t}\right)$ is nothing else but $L^{2}\left(\mathrm{~V}_{t \in I} \mathcal{B}_{t}\right)$, and $X_{t_{d}}$ is just $L^{1}\left(\mathrm{~V}_{t \in I} B_{t}\right)$. On the other hand, a subset of $L^{1}(\mathcal{B})$ is relatively compact in the $\sigma\left(L^{1}, L^{\infty}\right)$-topology if and only if it is uniformly integrable, so in this case Theorem 2 gives the known result on limits of martingales:

Theorem $2^{\prime}$. Let $\left\{f_{t}, \mathcal{B}_{t}, t \in I\right\}$ be a martingale defined on an interval $I$ of $\mathrm{R}$, open to the right. Then the following conditions are equivalent:

1) $\lim _{t \uparrow t_{d}} f_{t}$ exists in $L^{1}(\mathcal{B})$.

2) There exists an $f \in L^{1}\left(\vee_{t \in I} B_{t}\right)$ such that $f_{t}=E\left(f \mid B_{t}\right)$ for all $t \in I$.

3) The family $\left\{f_{t}: t \in I\right\}$ is uniformly integrable.

Example 5. Return to Example 3. If $\left\{f_{t}, \mathcal{B}_{t}, t \in I\right\}$ is an $\mathcal{H}$-valued martingale on an interval $I$ of $R$, then Theorem 2 holds. It should be noticed that since $\mathcal{H}$ is reflexive, the family $\left\{f_{t}: t \in I\right\}$ is weakly relatively compact if and only if it is uniformly integrable, i.e. if and only if the family of real integrable functions $\left\{\left\|f_{t}(\omega)\right\|: t \in I\right\}$ is uniformly integrable. (Here $\|\cdot\|$ denotes the norm in $\mathcal{H}$.)

\section{The case where $\boldsymbol{X}$ is weakly sequentially complete.}

In this section we shall see that the continuity of the map from $X_{1}{ }^{*}$ into $X^{*}$ defined previously is not needed when $X$ is weakly sequentially complete. This is the case in Example 1.

Theorem 3. Let $\left(X, H, X^{*}\right)$ be a CB-space and suppose that $X$ is weakly sequentially complete. Then if $H_{1}$ is a closed subspace of $H$, and Condition 1 is satisfied, $\left(X_{1}, H_{1}, X_{1}^{*}\right)$ is a sub-CB-space of $\left(X, H, X^{*}\right)$.

Proof. Put $X_{1}{ }^{\prime}=\left\{x \in X \mid\left\langle x, \bar{x}_{1}^{*}\right\rangle=0 \quad \forall x_{1}^{*} \in X_{1}^{*}\right\}$. Then $X_{1} \cap X^{\prime}=\{0\}$ since, by definition, $\left.\bar{x}_{1}{ }^{*}\right|_{H_{1}}=\left.x_{1}{ }^{*}\right|_{H_{1}}$, whence $\bar{x}_{1}{ }^{*}$ and $x_{1}{ }^{*}$ agree on $X_{1}$. Thus, if $x_{1} \in X_{1} \cap X_{1}^{\prime}$, then $\left\langle x_{1}, x_{1}^{*}\right\rangle=0$ for all $x_{1}^{*} \in X_{1}^{*}$ and so $x_{1}=0$.

Now suppose $x_{n}$ is a weak Cauchy sequence of elements of the space $X_{1} \oplus X_{1}^{\prime}(\oplus$ denotes algebraic direct sum),

$$
x_{n}=x_{1}{ }^{n}+x_{1}{ }^{n}, \quad \text { where } x_{1}{ }^{n} \in X_{1} \text { and } x_{1}{ }^{n} \in X_{1}{ }^{\prime} \text {. }
$$


Since $X$ is weakly sequentially complete, there is an element $x$ in $X$ such that

$$
\lim _{n \rightarrow \infty}\left\langle x_{n}, y^{*}\right\rangle=\left\langle x, y^{*}\right\rangle \text { for all } y^{*} \text { in } X^{*} \text {. }
$$

In particular, for every $x_{1}^{*} \in X_{1}^{*}$,

$$
\begin{aligned}
\lim _{n \rightarrow \infty}\left\langle x_{n}, \bar{x}_{1}{ }^{*}\right\rangle & =\lim _{n \rightarrow \infty}\left\langle x_{1}{ }^{n}+x_{1}{ }^{n}, \bar{x}_{1}{ }^{*}\right\rangle \\
& =\lim _{n \rightarrow \infty}\left\langle x_{1}{ }^{n}, x_{1}{ }^{*}\right\rangle=\left\langle x, \bar{x}_{1}{ }^{*}\right\rangle .
\end{aligned}
$$

Thus $x_{1}{ }^{n}$ is a weak Cauchy sequence in $X_{1}$, and hence it converges weakly to some $x_{1} \in X_{1}$. Now look at $x-x_{1}$, and let $x_{1}{ }^{*} \in X_{1}{ }^{*}$. Then

$$
\begin{aligned}
\left\langle x-x_{1}, \bar{x}_{1}^{*}\right\rangle & =\left\langle x, \bar{x}_{1}^{*}\right\rangle-\left\langle x_{1}, \bar{x}_{1}^{*}\right\rangle \\
& =\lim _{n \rightarrow \infty}\left\langle x_{1}{ }^{n}+x_{1}{ }^{\prime n}, \bar{x}_{1}{ }^{*}\right\rangle-\left\langle x_{1}, x_{1}{ }^{*}\right\rangle \\
& =\lim _{n \rightarrow \infty}\left\langle x_{1}{ }^{n}, x_{1}{ }^{*}\right\rangle-\left\langle x_{1}, x_{1}{ }^{*}\right\rangle=0,
\end{aligned}
$$

and hence $x-x_{1} \in X_{1}{ }^{\prime}$. We have proved: The weak limit (if it exists) of a sequence from $X_{1} \oplus X_{1}^{\prime}$ belongs to $X_{1} \oplus X_{1}{ }^{\prime}$. This implies that $X_{1} \oplus X_{1}{ }^{\prime}$ is closed in $X$ with respect to $\|\cdot\|$.

Furthermore, $H \ominus H_{1} \subset X_{1}{ }^{\prime}$. For let $y \in H \ominus H_{1}$, and let $x_{1}{ }^{*} \in X_{1}{ }^{*}$. Then

$$
\left\langle y, \bar{x}_{1}^{*}\right\rangle=\left(y, \varphi_{1}\left(x_{1}^{*}\right)\right)=0 \quad \text { since } \varphi_{1}\left(x_{1}^{*}\right) \in H_{1}
$$

(for definition of $\varphi_{1}\left(x_{1}{ }^{*}\right)$ see p. 228). Hence

$$
H=H_{1} \oplus\left(H \ominus H_{1}\right) \subset X_{1} \oplus X_{1}^{\prime},
$$

and since $H$ is dense in $X$, we conclude that

$$
X=X_{1} \oplus X_{1}^{\prime} \text {. }
$$

$X_{1}{ }^{\prime}$ is obviously closed in $X$ so by the closed graph theorem the projection $\bar{P}$ from $X$ onto $X_{1}$ along $X_{1}{ }^{\prime}$ is continuous. $\bar{P}$ is an extension of $P$ because $H_{1} \subset X_{1}$ and $H \ominus H_{1} \subset X_{1}^{\prime}$.

Remark. From Theorem 1 it now follows that the injection from $X_{1}{ }^{*}$ into $X^{*}$ is continuous.

\section{REFERENCES}

1. Nguyen-Xuan-Loc et E. Briem, Les martingales généralisées, C. R. Acad. Sci. Paris Sér. A-B 268 (1969), A 1107-A 1110.

2. J.-P. Vigier, Etude sur les suites infinies d'opérateurs hermitiens, Thèse, Univ. Genève, 1946. 